\title{
Décharge capillaire compacte et ultra-brève pour la lithographie UV extrême par projection
}

\author{
P. Choi ${ }^{1}$, I. Krisch ${ }^{1,2}$, J. Larour ${ }^{1}$, C. Dumitrescu ${ }^{1,3}$, M. Favre ${ }^{4}$, A. Chuvatin ${ }^{1}$, \\ J. Rous' ${ }^{1}$, C. Leblanc ${ }^{3}$ et A. Guilbert ${ }^{3}$ \\ ${ }^{1}$ Laboratoire de Physique des Milieux lonisés, UMR 7648 du CNRS, Ecole Polytechnique, \\ 91128 Palaiseau, France \\ 2 John Caunt Scientific Ltd., Oxford OX9 2QA, U.K. \\ ${ }^{3}$ EPPRA, XTEC, École Polytechnique, 91128 Palaiseau, France \\ ${ }^{4}$ Facultad de Fisica, Pontificia Universidad Catolica de Chile, Santiago 22, Chile
}

\begin{abstract}
Résumé : La lithographiẹ UV extrême (UVE) doit jouer un rôle majeur dans le système de nouvelle génération pour produire des composants micro-électroniques de dimension caractéristique inférieure à 100 nm. Une source efficace de rayonnement UVE dans la gamme 10-15 $\mathrm{nm}$ a été développée pour répondre aux demandes d'application pratique. Dans ce papier, nous présentons une source originale par décharge capillaire ultra brève assistée par effet de cathode creuse transitoire. L'utilisation de Xénon à basse pression permet de répondre à la contrainte en terme de longueur d'onde. Après avoir été initiée par l'effet de cathode creuse, la décharge part effectivement de l'axe du tube capillaire. Le canal de plasma qui en résulte est alors chauffé en utilisant une énergie stockée inférieure à $0,5 \mathrm{~J}$, jusqu'atteindre une température de $30 \mathrm{eV}$ dans un temps typiquement nanoseconde. Pendant les 5 ns que dure l'émission UVE, le plasma transitoire, au sein du tube dont le rapport d'aspect est très supérieur à 10 , émets principalement entre $10 \mathrm{~nm}$ et $20 \mathrm{~nm}$, avec une taille de source inférieure à $200 \mu \mathrm{m}$ en diamètre. Le spectre est formé par les raies Xe VII à Xe X. La très haute efficacité de conversion énergétique dans ce dispositif permet une extrapolation pour un fonctionnement répétitif.
\end{abstract}

La lithographie UV extrême (UVE) est un sujet d'actualité et beaucoup de sources sont proposées pour générer le rayonnement demandé, en particulier près de 13,5nm [1]. Cependant les sources basées sur des décharges sont peu souvent étudiées dans ce but. Nous présentons ici l'évaluation d'un dispositif à l'étude depuis plusieurs années $[2,3]$ pour le cas de la lithographie UVE. Compte tenu de la température de plasma atteinte sur l'axe dans les décharges capillaires rapides assistées par effet de cathode creuse, soit de 30 à $60 \mathrm{eV}$, on peut espérer obtenir des ions de forte charge. En examinant les transitions se situant dans la bande d'intérêt, on note que le xénon est un bon candidat comme gaz porteur de la décharge.

On a donc étudié systématiquement le fonctionnement de la décharge capillaire en présence d'un flux de xénon. Toutes les études systématiques [2,3] ont été répétées avec différentes tailles de cellule et différents diamètres internes de capillaire. Il est apparu que la combinaison d'une cellule rapide (capacité $\operatorname{lnF}$ ) et un petit capillaire (alumine, diamètre $0,8 \mathrm{~mm}$ ) permettait d'obtenir la meilleure efficacité de conversion dans la bande spectrale recherchée. Le fonctionnement est déclenché par un canon à plasma et assisté par effet de cathode creuse, c'est à dire qu'un faisceau d'électrons énergétiques parcourt le capillaire sur son axe, ionisant le gaz présent tout en préservant le milieu d'une ablation des parois. La préionisation rend possible le passage d'un fort courant (quelques kA) dans ce canal, et le chauffage du plasma. Un spectrographe UV compact, à réseau gravé sous incidence rasante, est placé sur l'axe côté anode et trois spectres (3-30nm) sont pris pendant le tir, avec une fenêtre d'ouverture de $3 \mathrm{~ns}$ et une séparation de 10ns.

On a ainsi répété tous les tests électriques et optiques $[2,3]$ pour trouver loptimum de pression de Xénon $(0,4$ mbar) comme on le voit clairement sur la Figure 1, et affiner l'identification des spectres. Les raies du Xénon X sont clairement présentes et prouvent l'existence dans le plasma chaud des ions Xénon 6 fois chargés à 9 fois chargés. Les raies de Xe IX et Xe X dominent le spectre mais le manque de données spectroscopiques $[4,5]$ limite cette dernière conclusion. Les mesures de taille de plasma par caméra fentefil [2] donnent un diamètre de $100 \mu \mathrm{m}$ au démarrage, montant à $300 \mu \mathrm{m}$ lors du maximum d'émission.

Il est nécessaire pour parler d'applicabilité de chiffrer le flux émis par la source. Pour ceci nous avons placé un jeu de diodes PIN absolues calibrées (surface $0,06 \mathrm{~mm}^{2}$, réponse $0,3 \mathrm{~ns}$ ), respectivement sans filtre, avec un filtrage $\mathrm{Al}$ 0,75 micron et un filtrage $\mathrm{Al}$ 1,5micron. L'écran d'aluminium coupe une bande jusqu'à $17 \mathrm{~nm}$ et on peut raisonnablement estimer le flux UVE par différence entre le flux total et le flux écranté. La Figure 2 montre les trois signaux ramenés à un flux énergétique et le flux UVE obtenu par différence. On voit que celui ci se situe au maximum à $14 \mathrm{~kW} / \mathrm{sr}$ et la durée de l'impulsion est inférieure à $15 \mathrm{~ns}$, ceci pour une énergie électrique rendue à la cellule de $0,2 \mathrm{~J}$ oule par tir.

En conclusion de cette étude sur une cellule non optimisée, il est possible d'obtenir une émission centrée sur la bande d'intérêt en Lithographie UVE. Il serait possible d'augmenter notablement le taux de répétition, actuellement de l'ordre du $\mathrm{Hz}$ à condition de contrôler l'échauffement de la paroi interne du capillaire exposée également au flux photonique et d'augmenter la puissance de l'alimentation. Elle se 
compare favorablement aux autres sources par décharge électrique rapide [6,7] du point de vue de la compacité. Les partenaires étudient les options d'un développement vers l'application.

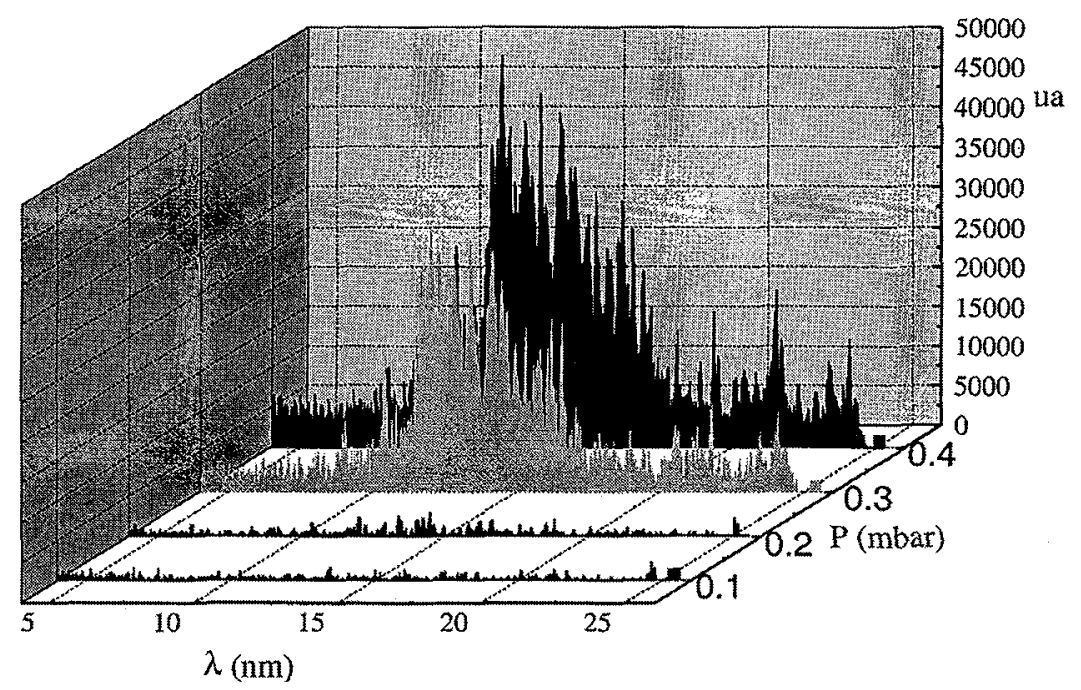

Figure 1 : Dépendance en pression du spectre de Xénon émis par la décharge

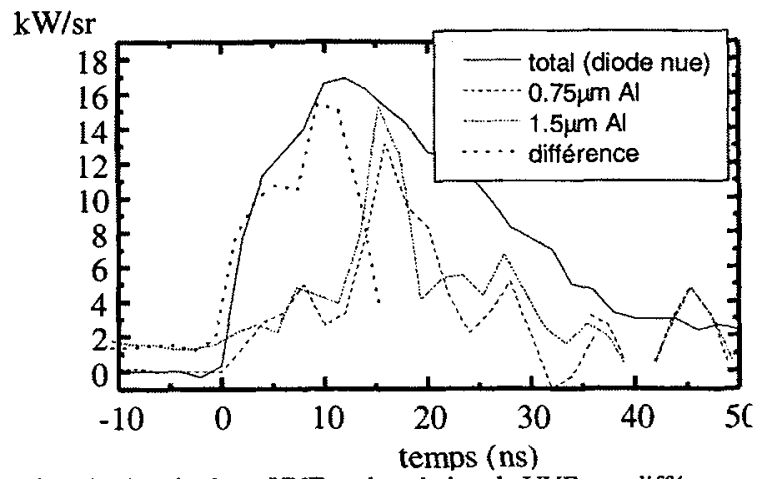

Figure 2 : Signal optique total, dans les bandes hors UVE et dans la bande UVE par différence

\section{Remerciements}

Travail soutenu par la Commission Européenne sur contrat TMR ERBFMRXCT980186 (FACADIX) et par un programme d'échange bilatéral CNRS-CONICYT. http://www.facadix.net

\section{References}

1. J.E. Bjorkholm, Intel Technology Journal Q3 198 (1998), 1-8.

2. I. Krisch, P. Choi, J. Larour, J.Rous, C. Dumitrescu, A.Chuvatin, S. Zakharov, C. LeBlanc et M. Favre, cette conférence

3. P. Choi and M. Favre, Review Scientific Instr. 69 (1998), 3118.

4. J. Blackburn, P.K. Carroll, J. Costello, G. O'Sullivan, J. Opt. Soc. Am 73, 1325 (1983)

5. V. Kaufman, J. Sugar, J.L. Tech, J. Opt. Soc. Am 73, 691 (1983)

6. M. McGeoch, Appl. Opt. 37, 1651 (1998)

7. M.A. Klosner, W.T. Silfvast, Opt. Lett. 23, 1609 (1998) 